\section{DESIGN OF DUAL-MODE DUAL-BAND PHOTONIC CRYSTAL BANDPASS \\ FILTERS FOR TERAHERTZ COMMUNICATION APPLICATIONS}

Ozgur Onder Karakilinc ${ }^{1}$ and Mehmet Salih Dinleyici ${ }^{2}$

${ }^{1}$ Department of Electrical and Electronics Engineering, Pamukkale

University, 20070 Denizli, Turkey; Corresponding author: okarakilinc@pau.edu.tr

${ }^{2}$ Department of Electrical and Electronics Engineering, Izmir Institute of Technology, 35437 Urla, Izmir, Turkey

Received 30 January 2015

ABSTRACT: In this study, Photonic Crystal (PhC) dual-mode dualband bandpass filter is designed and its transmission characteristics are investigated for various configurations. PhC resonator structure is formed by a point defect microcavity that is equipped with one large and three smaller auxiliary perturbation rods. Degenerate modes at each band may also be excited by changing the structure properties of the perturbation. Plane wave expansion method and Finite Difference Time-Domain method are used to analyze the device characteristics. Proposed dual-band dual-mode PhC filter structure can effectively be used for terahertz communication applications. (C) 2015 Wiley Periodicals, Inc. Microwave Opt Technol Lett 57:1806-1810, 2015; View this article online at wileyonlinelibrary.com. DOI 10.1002/mop.29196

Key words: degenerate mode; dual band; dual mode; filter; photonic crystal

\section{INTRODUCTION}

Photonic crystals (PhCs) are artificial materials which include a periodic arrangement of dielectric medium and display photonic band gaps depending on their geometry and refractive index profiles. Because of the unique properties on the light guidance, $\mathrm{PhCs}$ have become as indispensable tool for light wave manipulation. Several functions like filters, couplers, and multiplexers have already been designed using localized defect modes in PhC [1]. Yet, the need for high speed and high data rate communication systems leads to an increased attention to terahertz $(\mathrm{THz})$



Figure $12 \mathrm{D} \mathrm{PhC}$ structure. [Color figure can be viewed in the online issue, which is available at wileyonlinelibrary.com]

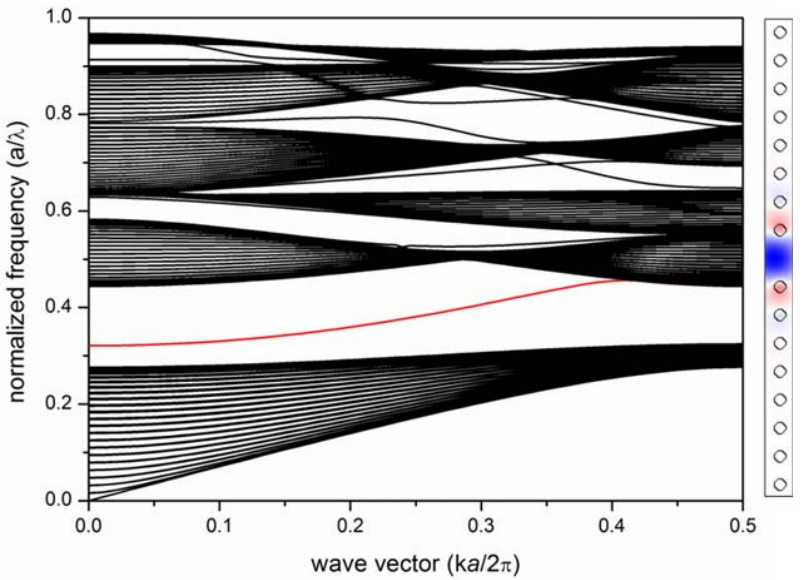

Figure 2 TE (TM, according to Meep) band structure of square PhC with a line defect waveguide and propagated mode profile. [Color figure can be viewed in the online issue, which is available at wileyonlinelibrary.com]

frequencies [2-4]. The micrometers order lattice constant of $\mathrm{PhC}$ is very convenient for conventional manufacturing methods and applicable for applications in $\mathrm{THz}$ range [5]. Therefore, intense research force has been dedicated to the $\mathrm{PhC}$ structures that exhibit various functions necessary in that range [5-7]. Along with the progress in $\mathrm{THz}$ communication technologies, filters for multiple frequency bands are highly needed for spectral management in systems with compact design [8]. In this article, dual-mode dual-band $\mathrm{PhC}$ bandpass filter is proposed and its transmission characteristics are investigated for various configurations. Degenerate modes of $\mathrm{PhC}$ microcavity constructed by a point defect placed at the corner of the line defect waveguide shown in Figure 1 can be splitted by modifying the auxiliary perturbation rods. Exploiting degenerate mode splitting in PhC cavity, single band filter characteristics have been obtained by recent studies $[9,10]$. Deviating from that studies, in this work, we propose $\mathrm{PhC}$ compact bandpass filter exhibiting dual-mode dual-band characteristic. Plane wave expansion method (Mpb) and Finite Difference Time-Domain method (Meep), which are developed by Massachusetts Institute of Technology (MIT) photonic research group, are used to analyze the device characteristics [11].

\section{PROPOSED PhC MICROCAVITY STRUCTURE}

In this study, two-dimensional (2D) square lattice $\mathrm{PhC}$ structure with alumina rods in air substrate is considered. As simulation geometry depicted in Figure 1, there is a line defect $\mathrm{PhC}$ waveguide with a microcavity having radius $r_{\mathrm{d}}$ that is placed at the bend. $\mathrm{PhC}$ dimensions are $25 a \times 25 a$ along the $x$ and $y$ directions of $2 \mathrm{D} \mathrm{PhC}$. The lattice constant " $a$ " is in unit of $\mu \mathrm{m}$. If the normalized values are used, then all the values in the simulation are normalized with $\mu \mathrm{m}$ and frequency is also normalized to $a / \lambda$. All other lengths are expressed in terms of " $a$ " that is set to $400 \mu \mathrm{m}$ which is very convenient for fabrication $[5,12]$.

The radius of the rods is chosen $0.2 a$ and the dielectric constant of alumina is $8.9(\varepsilon)$. Propagating modes are obtained from the band diagram of line defect $\mathrm{PhC}$ as shown in Figure 2 with a red line. Fourier transformed propagated mode profile is computed by MATLAB and applied to the input of waveguide as a plane wave source. Thus, single guided mode (eigenmode) excitation is guaranteed. The transmitted mode fields are detected with a line detector at the output. Throughout the simulations, 


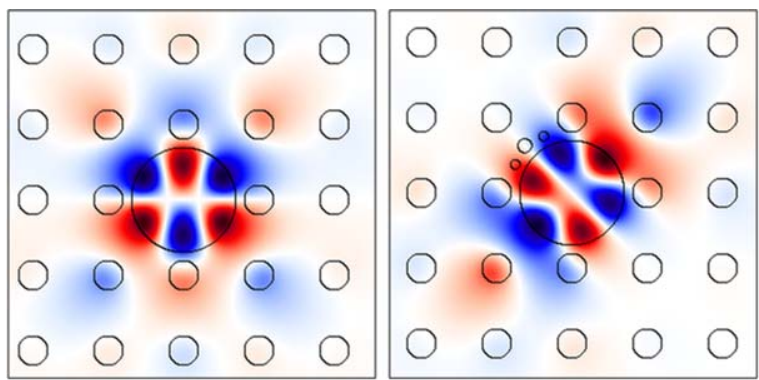

(a)

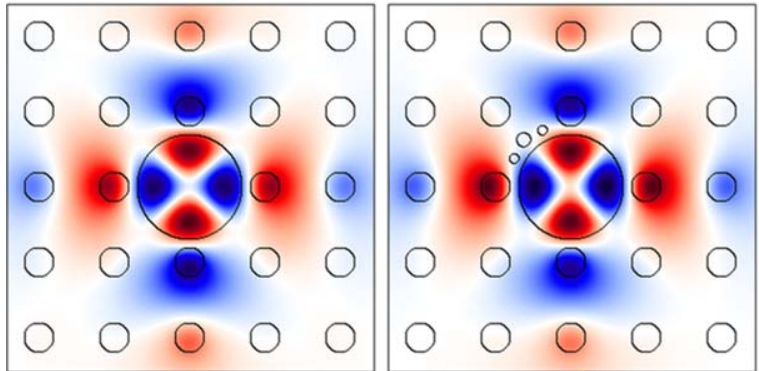

(c)

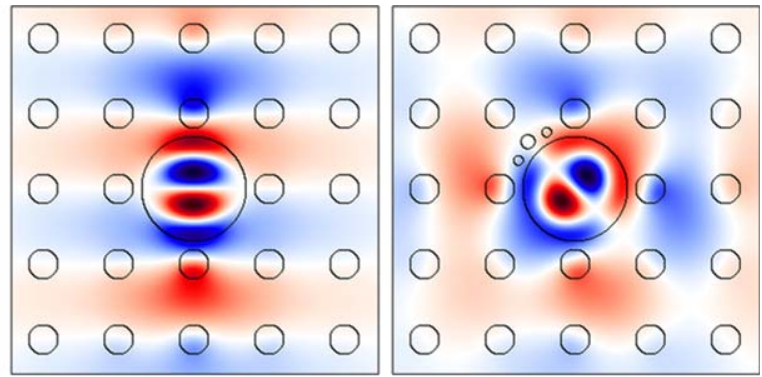

(b)

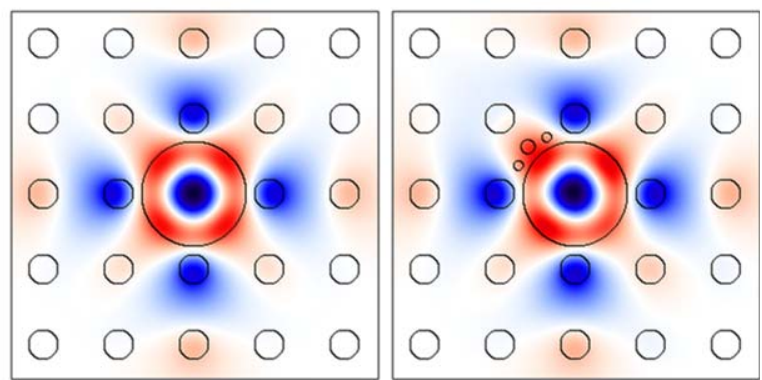

(d)

Figure 3 Doubly degenerate (left) and coupled (right) (a) hexapole and (b) dipole modes for $0.29 \mathrm{THz}$ and $0.324 \mathrm{THz}$, respectively. Non-degenerate (left) and coupled (right) (c) quadrupole and (d) monopole mode for $0.261 \mathrm{THz}$ and $0.264 \mathrm{THz}$, respectively. [Color figure can be viewed in the online issue, which is available at wileyonlinelibrary.com]

the field is assumed as TE polarized (TM, according to Meep) where the electric field vector is perpendicular to the plane of $\mathrm{PhC}$.

$\mathrm{PhC}$ microcavity resonator in this study has $\mathrm{C}_{4 v}$ symmetry where this structure does not change by $90^{\circ}$ rotation. Hence, the cavity modes can be classified as doubly degenerate and nondegenerate in accordance with the symmetry properties. The modes are called nondegenerate if their shapes are invariant under the rotation operator [13]. There are four modes revealed in the PhC microcavity. The two modes are doubly degenerate and the other two are nondegenerate if the radius of microcavity rod is about $0.7 a$ [1]. Doubly degenerate hexapole and dipole resonance modes at the frequency of the lower band $(0.29 \mathrm{THz})$ and the upper band $(0.324 \mathrm{THz})$ are shown in Figure 3(a) and quality factor, $Q$ of the microcavity is calculated as $0.3 \times 10^{6}$ and $0.6 \times 10^{6}$, respectively. To excite the degenerate modes in each band simultaneously, a novel perturbation structure is needed and investigated in this study. There are three small perturbation rods at upper left corner of the microcavity. Their names are $r_{\text {pmid }}$ and $r_{\mathrm{p}}$ (Fig. 4). Apart from the other perturba-

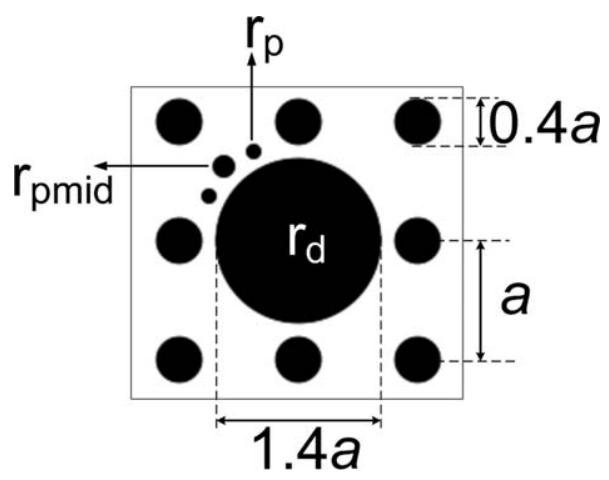

Figure 4 Dual-mode dual-band microcavity tion rods, the middle rod has different radius. The refractive index of all perturbation rods is the same as the $\mathrm{PhC}$ rods. Exact position of $r_{\text {pmid }}$ and $r_{\mathrm{p}}$ are $(0.625,0.625),(-0.375,0.75)$, $(-0.75,0.375)$ points with respect to the center of microcavity rod $r_{\mathrm{d}},(0,0)$ in $(x, y)$ plane. The degenerate modes appear by applying the perturbation rods configuration as seen in Figures 3 (a) and 3(b). The quality factor of coupled hexapole and dipole modes can be calculated as $1.6 \times 10^{6}$ for $0.29 \mathrm{THz}$ and $0.15 \times$ $10^{6}$ for $0.324 \mathrm{THz}$ with perturbation $r_{\mathrm{p}}=0.07 a, r_{\mathrm{pmid}}=0.1 a$. Besides, field distributions of nondegenerate quadrupole and monopole modes persist with the perturbation in Figures 3(c) and $3(\mathrm{~d})$.

\section{DUAL-BAND FILTER CHARACTERISTICS}

The filter characteristics are obtained for various perturbation configurations as shown in the Figures $5(\mathrm{a})-5(\mathrm{~g})$. There is no transmission in the line defect waveguide when using the microcavity without perturbation rods as depicted in Figure 5(a). According to Figure 5(b), the transmission spectrum is dramatically changed by introducing a rod $r_{\text {pmid }}=0.07 a$ at the upper left corner of microcavity and between two $\mathrm{PhC}$ rods. The band at the frequency of $0.29 \mathrm{THz}$, which is called lower band, is transmitting, but the degenerate modes are splitted as two peaks. Meanwhile, the transmissivity of upper band, at the frequency of 0.324 is increased, but the reflection level is more than -5 $\mathrm{dB}$. The transmission begins at the upper band with the larger value of $r_{\text {pmid }}$ as $0.1 a$; however, the degenerate modes in the lower band are overcoupled which means the band is deformed. A degenerate mode in the upper band is not excited in this case as seen in Figure 5(c). If only $r_{\mathrm{p}}$ perturbation is applied as in Figure 5(d), the lower band is turned to transmit; however, the upper band transmission remains at the level of $-5 \mathrm{~dB}$. Similarly to Figure 5(c), transmission of the upper band and mode splitting can be seen in Figure 5(e) using the larger value of $r_{\mathrm{p}}=0.1$. By proposing the novel perturbation structure as seen 

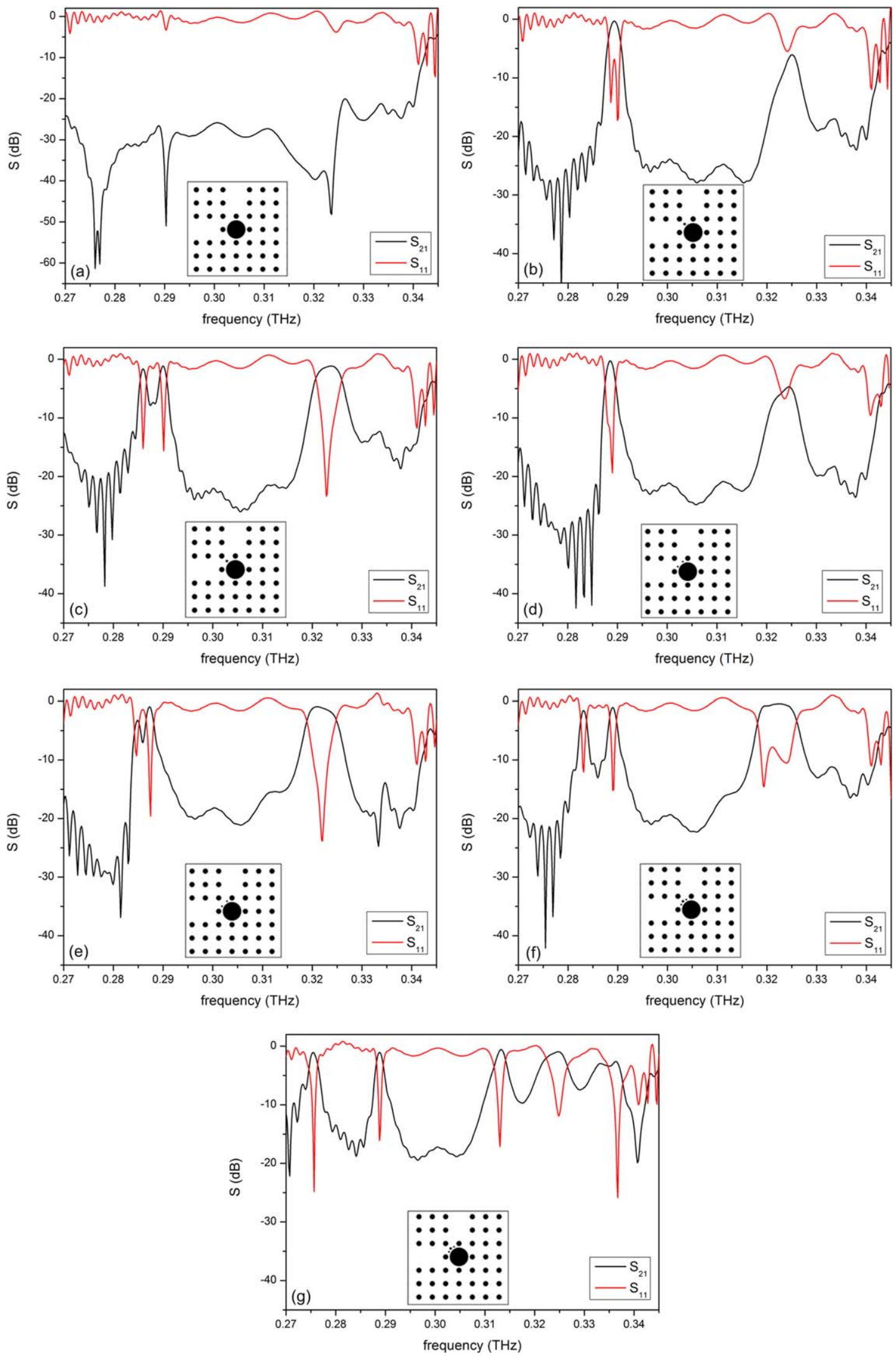

Figure 5 (a) Transmission and reflection spectrum of microcavity with $r_{\mathrm{p}}=0$ and $r_{\text {pmid }}=0$ (b) $r_{\mathrm{p}}=0$ and $r_{\text {pmid }}=0.07 a$ (c) $r_{\mathrm{p}}=0$ and $r_{\text {pmid }}=0.1 a$ (d) $r_{\mathrm{p}}=0.07 a$ and $r_{\mathrm{pmid}}=0$ (e) $r_{\mathrm{p}}=0.1 a$ and $r_{\mathrm{pmid}}=0$ (f) $r_{\mathrm{p}}=0.07 a$ and $r_{\mathrm{pmid}}=0.1 a(\mathrm{~g}) r_{\mathrm{p}}=0.07 a$ and $r_{\mathrm{pmid}}=0.15 a$. [Color figure can be viewed in the online issue, which is available at wileyonlinelibrary.com] 


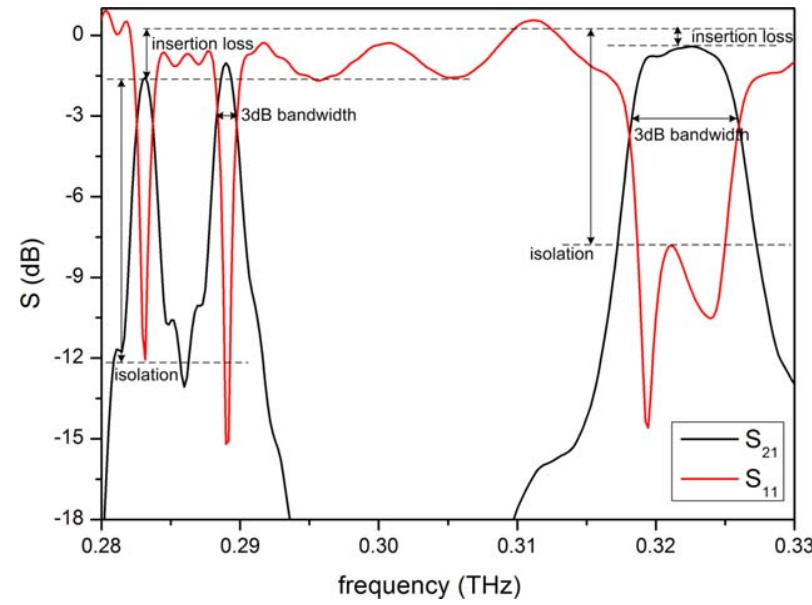

Figure 6 Transmission parameters of the lower and upper band with $r_{\mathrm{p}}=0.07 a$ and $r_{\text {pmid }}=0.1 a$. [Color figure can be viewed in the online issue, which is available at wileyonlinelibrary.com]

in Figure 5(f), dual-mode dual-band bandpass filter is obtained by splitting degenerate modes at the both bands. In the last configuration in Figure 5(f), the mode in the upper band is splitted using the perturbation of $r_{\mathrm{p}}=0.07 a$ and $r_{\text {pmid }}=0.1 a$. Degenerate modes in the lower band as well as the upper band are overcoupled with a further increase of $r_{\text {pmid }}$ as presented in Figure $5(\mathrm{~g})$. According to the simulation results, the isolation is higher

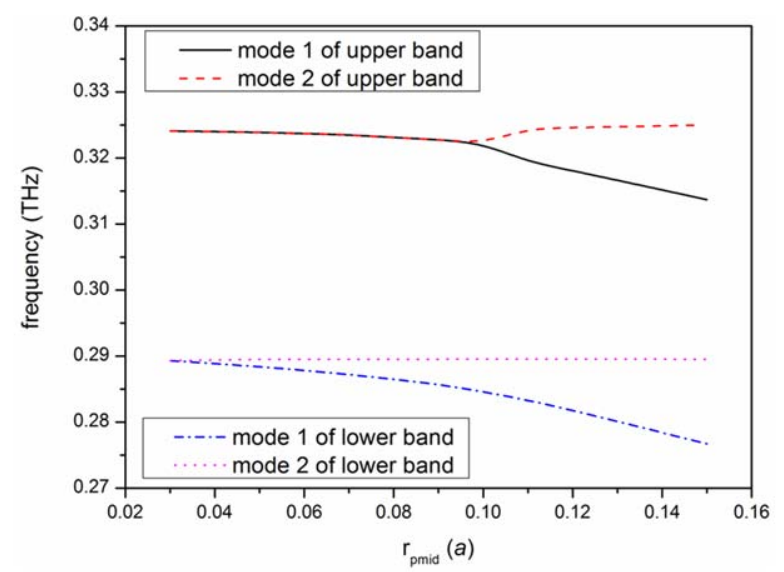

(a)

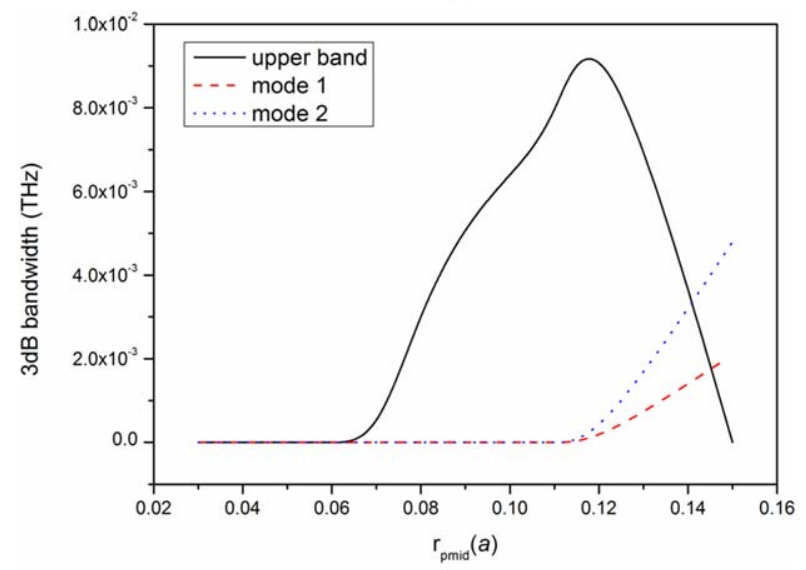

(c) than $10 \mathrm{~dB}$ and the insertion losses are 1.55 and $1.02 \mathrm{~dB}$ as shown in Figure 6. Three-decibel bandwidth is also $0.0011 \mathrm{THz}$ and $0.0014 \mathrm{THz}$ for the left and right modes at lower band, respectively. In contrast to the lower band, the upper band presents larger bandpass filter characteristics with the isolation is higher than $7 \mathrm{~dB}$ and the insertion losses is $0.41 \mathrm{~dB}$ and $3-\mathrm{dB}$ bandwidth is $0.0075 \mathrm{THz}$. The upper band exhibits better flat spectral characteristic than the lower band. Fractional bandwidth $(\Delta f / f)$ is calculated as $\% 0.3$ and $\% 0.4$ for each mode of the lower band and \% 2.3 for the upper band. Fractional bandwidth can be tuned by perturbation rods.

\section{OPTIMUM PARAMETERS FOR DUAL-MODE DUAL-BAND FILTER}

Various forms of the $\mathrm{PhC}$ microcavity structures have been investigated by changing radius of the perturbation rods, $r_{\text {pmid }}$ $0.03 a$ to $0.15 a$ while the $r_{\mathrm{p}}$ is set to $0.05 a$. The lower band at frequency $0.29 \mathrm{THz}$ is excited before the upper band at frequency $0.324 \mathrm{THz}$. The critical coupling value of $r_{\text {pmid }}$ is $0.03 a$ and $0.10 a$ for the lower band and the upper bands, respectively. At the values $0.10 a$ of the $r_{\text {pmid }}$, then the upper band is to split as shown in Figure 7(a). Adjusting the middle perturbation rods radius $r_{\text {pmid }}$ as $0.11 a$, both bands can be simultaneously excited. Spectral shift between the degenerate mode frequencies in each band is increased by $r_{\text {pmid. }}$. Transmission coefficient $\left(S_{21}\right)$ and bandwidth of the both bands are also increased by $r_{\text {pmid }}$. But for the larger values of $r_{\text {pmid }}$, the upper band is over coupled because of the decreasing $S_{21}$ as seen in Figure 7(b). Besides,

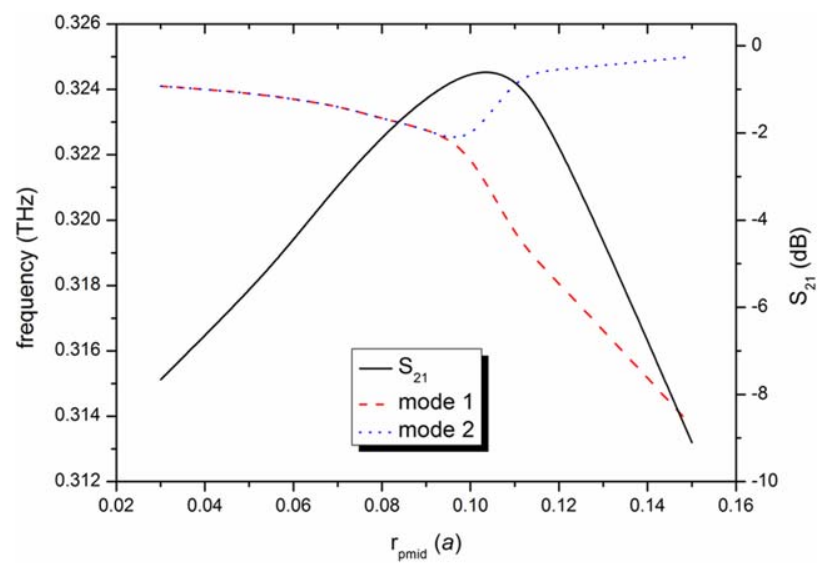

(b)

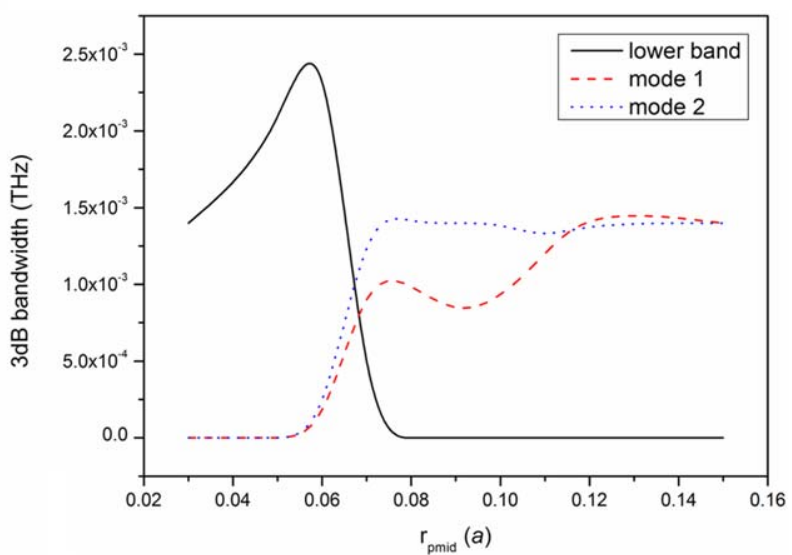

(d)

Figure 7 Band splitting properties for $r_{\text {pmid }}$ variation $\left(r_{\mathrm{p}}\right.$ is set to $\left.0.05 a\right)$. [Color figure can be viewed in the online issue, which is available at wileyonlinelibrary.com] 
when the splitting of modes is increased by $r_{\text {pmid }}$, the bandwidth of modes is increased as depicted in Figures 7(c) and 7(d). Additionally, resonance mode frequency can be tuned with exploiting nonlinearity in $\mathrm{PhC}$ cavity. If refractive index of the microcavity rod is increased about $\% 1$, the band frequency shifts about $0.003 \mathrm{THz}$ to lower frequency part of the spectrum. Apart from the index change of microcavity rods, the transmission spectrum is not affected by the index variation of perturbation rods. The modes can be easily tuned by perturbation rod structure and/or nonlinear index variation of microcavity rod.

\section{CONCLUSION}

In this article, we have proposed $\mathrm{PhC}$ microcavity structures having perturbation rods that show dual-mode, dual-band filter characteristics with narrow bands, high isolation, low insertion loss, and high quality. The degenerate modes at each band can be activated concurrently with the perturbation rods with $r_{\mathrm{p}}=0.05$ and $r_{\text {pmid }}=0.11$. The upper band presents flat spectral characteristics as bandwidth is $0.0076 \mathrm{THz}$ with fractional bandwidth \% 2.3 at $0.322 \mathrm{THz}$. The lower band becomes overcoupled with the larger value of $r_{\text {pmid. }}$. However, it can be used as a narrow band filter with the bandwidth of $0.0012 \mathrm{THz}$ and $0.0014 \mathrm{THz}$ and fractional bandwidth of \% 0.4 for each mode of $0.283 \mathrm{THz}$ and $0.289 \mathrm{THz}$, respectively. Although the transmission spectrum is dramatically affected by changing the perturbation rod structure (dimension), changing the refractive index of the perturbation rods does not produce similar effects. Additionally, the band frequency is shifted about $0.003 \mathrm{THz}$ to lower frequency part of the spectrum by the refractive index of the microcavity rod is increased about $\% 1$. This study may contribute to the development of functional devices for wireless communication application at $\mathrm{THz}$ range.

\section{REFERENCES}

1. R.D. Meade, J.D. Joannopoulos, S.G Johnson, and J.N Winn, Photonic crystals: Molding the flow of light, 2nd ed., Princeton University Press, New Jersey, 2008.

2. T. Kleine-Ostmann and T. Nagatsuma, A review on terahertz communications research, J Infrared Millimeter Terahertz Waves 32 (2011), 143-171.

3. H. Shirai, K. Ishii, H. Miyagawa, S. Koshiba, S. Nakanishi, and N. Tsurumachi, Efficient terahertz emission, detection, and ultrafast switching using one-dimensional photonic crystal microcavity, J Opt Soc Am B 31 (2014), 1393-1401.

4. Z. Wu, A. Young, M. Gehm, and H. Xin, Investigation of several terahertz electromagnetic band gap structures, Microwave Opt Technol Lett 52 (2010), 678-686.

5. S. Kirihara, Fabrication of photonic crystal cavities for terahertz wave resonations, Nanofabrication, 2011.

6. D. Yang, T. Li, L. Rao, S. Xia, and L. Zhang, Terahertz functional devices based on photonic crystal and surface plasmon polaritons, Terahertz Sci Technol 5 (2012), 131-143.

7. L. Rao, D. Yang, and Z. Hong, Guiding terahertz wave within a line defect of photonic crystal slab, Microwave Opt Technol Lett 54 (2012), 2856-2858.

8. Q. Huang, X. Zhang, J. Xia, and J. Yu, Dual-band optical filter based on a single microdisk resonator, Opt Lett 36 (2011), 4494-6.

9. C. Chun-ping, T. Anada, S. Greedy, T.M. Benson, and P. Sewell, A novel photonic crystal band-pass filter using degenerate modes of a point-defect microcavity for terahertz communication, Microwave Opt Technol Lett 56 (2014), 792-797.

10. A. Daraei and F. Khozeymeh, Investigation on mode splitting and degeneracy in the L3 photonic crystal nanocavity via unsymmetrical displacement of air-holes, Int J Eng Sci 2 (2013), 146-150.

11. A.F. Oskooi, D. Roundy, M. Ibanescu, P. Bermel, J.D. Joannopoulos, and S.G. Johnson, Meep: A flexible free-software package for electromagnetic simulations by the FDTD method, Comput Phys Commun 181 (2010), 687-702.

12. M.J. Fitch and R. Osiander, Terahertz waves for communications and sensing, Johns Hopkins APL Tech Dig 25 (2004).

13. S. Kim and Y. Lee, Symmetry relations of two-dimensional photonic crystal cavity modes, IEEE Int J Quantum Electron 39 (2003), 1081-1085.

(C) 2015 Wiley Periodicals, Inc.

\section{A DUAL-BAND COMPACT PRINTED MONOPOLE ANTENNA USING MULTIPLE RECTANGLE-SHAPED DEFECTED GROUND STRUCTURES AND CROSS-SHAPED FEED LINE}

\author{
Sanjeev Kumar and Raghuvir Tomar \\ Department of Electronics and Communication Engineering, The \\ LNM Institute of Information Technology, Jaipur 302031, India; \\ Corresponding author: rtomar@Inmiit.ac.in, tomarlnmiit@gmx.com
}

Received 30 January 2015

\begin{abstract}
A compact printed monopole antenna with dual-band operation at $1.8 \mathrm{GHz}$ (GSM Band) and $5.2 \mathrm{GHz}$ (Wi-Fi Band) is presented. The antenna consists of a modified $U$-shaped radiating patch with a triple rectangular defect in the ground plane. The antenna is fed by a cross-shaped feed line, which helps in realizing the dual-band operation. The antenna has been designed using RO4350 substrate. The design size achieved is $19 \times 28 \mathrm{~mm}^{2}$. An impedance bandwidth of about $32 \%$ at $1.8 \mathrm{GHz}$ and about $29 \%$ at $5.2 \mathrm{GHz}$ for $10 \mathrm{~dB}$ return loss has been achieved. A size reduction of about $12 \%$ in comparison with a similar antenna without defected ground structures has been achieved. The proposed antenna has been fabricated and tested, and a good agreement between simulated and measured results is seen. Acceptable E-plane and $H$-plane radiation patterns have been observed through simulations, and a peak antenna gain of about $2.8 \mathrm{dBi}$ has been achieved. (C) 2015 Wiley Periodicals, Inc. Microwave Opt Technol Lett 57:1810-1813, 2015; View this article online at wileyonlinelibrary.com. DOI 10.1002/mop.29195
\end{abstract}

Key words: defected ground structure; dual-band; impedance bandwidth; microstrip; Wi-Fi; GSM

\section{INTRODUCTION}

Compact dual-band microstrip antennas are much in demand nowadays, as such antennas facilitate integration of different radio modules into the same piece of equipment [1]. The use of defected ground structures (DGSs) is one currently popular method used to reduce the design size of microstrip antennas (e.g., [2-11]). This article proposes a new symmetrical printed monopole antenna using multiple rectangular DGSs, for dualband operation at GSM and Wi-Fi bands. The presence of the DGS perturbs the current distribution in the ground plane, and thus, modifies the equivalent transmission line parameters over the defected regions [2-4]. Put differently, the DGS uses its slow-wave characteristics to reduce the size of the design. In practical terms, the use of the DGS enhances the antenna bandwidth by an amount that depends on the shapes, the locations, and the dimensions of the defects [5-11].

In the design reported herein, the use of the multiple rectangular-shaped DGSs is made to increase the bandwidth of the antenna, and to reduce the design size. A cross-shaped feed line is used to achieve the dual-band operation. The proposed structure, shown in Figure 1, resonates at 1.8 and 5.2 GHz. The design dimensions have been optimized using the HFSS 